\title{
Emotional Decision-Makers and Anomalous Attitudes towards Information*
}

\author{
Francesca Barigozzi ${ }^{\dagger}$ and Rosella Levaggi ${ }^{\ddagger}$
}

September 2008

\begin{abstract}
We use a simple version of the Psychological Expected Utility Model (Caplin and Leahy, QJE, 2001) to analyze the optimal choice of information accuracy by an individual who is concerned with anticipatory feeling. The individual faces the following trade-off: on the one hand information may lead to emotional costs, on the other the higher the information accuracy, the higher the efficiency of decision-making.

We completely and explicitly characterize how anticipatory utility depends on information accuracy, and study the optimal amount of information acquisition. We obtain simple and explicit conditions under which the individual prefers no-information or partial information gathering. We show that anomalous attitudes towards information can be more articulated than previously thought.
\end{abstract}

JEL Classification: D81; D83.

Key Words: Psychological expected utility, Information gathering, Bayesian updating.

A previous, different version of the paper circulated with the title "Emotional Decision-Makers and Information Gathering". The authors are grateful to Giacomo Calzolari, Louis Eeckhoudt, Claudia Scarani, Peter Sivey, Rani Spiegler, Claudio Zoli and seminar participants in Bologna, Konstanz, Marseille, Padova, Roma, Siena and Toulouse for helpful comments and discussions.

Department of Economics, University of Bologna, P.zza Scaravilli 2, 40126 Bologna (Italy). E-mail: francesca.barigozzi@unibo.it

Đepartment of Economics, University of Brescia, Via S. Faustino, 74b, 25122 Brescia (Italy). E-mail: levaggi@eco.unibs.it 


\section{Introduction}

Patients may be aware that they are at risk of high cholesterol or high blood pressure and that these health conditions can have serious consequences in the future. However, since perfect knowledge can lead to anxiety, full resolution of uncertainty may not be the best choice. Investors may fear their portfolio choices are not appropriate, and this could imply bad financial consequences in future periods. Taking into account the emotional effects of information acquisition, the optimal amount of information investors should acquire is not obvious. People may suspect their partner is having an affair. If the suspicion turns out to be founded, their future will be seriously compromised. The famous saying 'What you don't know won't hurt you' illustrates that full information learning is not always the preferred choice.

In standard decision theory, if information has decision-making value it is welfare improving, if it does not, it simply becomes irrelevant. However, this usual conjecture that more (free) information is preferred to less might not be valid when decision-makers' well-being also depends on anticipatory feelings. In the motivating examples above, decision-makers (DMs henceforth) face a risk that leads to physical negative consequences in the future and, in the present, can bring about anticipatory feelings as fear or discomfort. Whenever DMs fear bad outcomes, information makes discomfort rise such that anomalous attitudes towards information acquisition can occur in the form of complete or partial information avoidance behavior. This is true also when information has a decision-making value, as in the examples above, and as is widely documented in the case of health behavior. For example, according to medical evidence, in many situations patients avoid free information that could be used to take better decisions (see Jacobsen et al. 1997, Lerman et al. 1994 and 1998, Quaid and Morris 1993). ${ }^{1}$

The aim of this work is to investigate information gathering by emotional DMs. To do so we use a simple version of the Psychological Expected Utility (PEU) model proposed by Caplin and Leahy (2001). In the PEU model anticipatory utility is modeled as utility derived from the DMs' expectations concerning their future such that the DMs' well-being is not only defined over physical outcomes as in standard analysis, but also over beliefs about physical outcomes. What is crucial for our purpose is attitude towards information: in the PEU model DMs can

\footnotetext{
${ }^{1}$ For example, Lerman et al. (1998) demonstrated that $46 \%$ of subjects whose blood was tested for genetic mutations refused to receive the test results despite the fact that the test results indicated whether or not these subjects were susceptible to breast cancer later in life. By knowing more precise information on their risk of cancer patients would have been able to take better prevention measures.
} 
be averse or not to information. When they are information-averse they suffer if information is disclosed. In other words, information-averse DMs dislike bad news more than they like good news.

In our setting, if the DM remains ignorant he pays the cost of selecting an inefficient action. In fact, physical utility depends both on the state of nature and on a specific action that, in order to be most effective, has to be state-contingent. In addition, since the DM derives utility directly from his beliefs, he must also consider how the information he gathers will affect those beliefs. In the model the DM chooses the amount of information by selecting the accuracy of a signal. Thus, any level of information acquisition is admissible: the DM can decide to remain completely ignorant or, by searching for information from different sources, he can choose different amounts of information up to full information. ${ }^{2}$

By considering the possibility of searching for information characterized by different content precision, we address in details the trade-off between the physical benefits and emotional costs of information for DMs who are information-averse. We completely and explicitly characterize how anticipatory utility depends on information accuracy, and study the optimal amount of information acquisition. As it will be further specified below, our results complement and extend previous ones showing that anomalous attitudes towards information can be more frequent and more articulated than previously thought. Moreover, since our simple and transparent framework yields explicit solutions for the conditions under which anomalous attitudes towards information occur, it can be a useful tool for analyzing and interpreting decision-making and strategic interaction in those contexts where anticipatory feelings are relevant.

More in details, we show that anticipatory utility can be non-monotone in information accuracy. Depending on the interplay between the degree of the DM's information aversion and the level of uncertainty (which affects the cost of an inefficient action) the DM can prefer no information, partial information or full information acquisition.

To understand the interaction between information aversion and the level of uncertainty it is useful to notice that information aversion (implying concavity of the anticipatory utility

\footnotetext{
${ }^{2}$ For example, patients could consider some partially informative facts such as their family medical history, or they could look for some information concerning the probability of being high-risk conditional on their specific lifestyle. Whereas a medical test would correspond to a perfect signal. Investors could read financial newspapers to obtain information on financial market performance. Consulting their financial expert, instead, would be equivalent to solve all the uncertainty on their portfolio return. Distrustful partners could look for circumstantial evidence such as ambiguous messages on mobile phones. Otherwise, by employing a personal investigator to spy on their partner their could obtain irrefutable evidence.
} 
function) always induces discomfort for information acquisition when no action is available. Thus, discomfort from information systematically induces the DM not to acquire any information when information has no decision-making value. On the other hand, better actions may offset the effect of a bad signal on beliefs so that the DM ends up with an ex-post payoff higher than when he remains ignorant (in such a case no emotional costs from information arise even though the DM is information averse). When instead unfavorable updated beliefs due to bad news have a stronger impact than better choices, the DM ends up with an ex-post payoff lower than when he stays ignorant. From an ex-ante view point, in the latter case the anticipatory utility associated with information acquisition is lower than the payoff of remaining ignorant and the DM experiments discomfort from information even though information has decision-making value.

For anomalous behavior regarding information acquisition (i.e. full or partial avoidance) it is necessary that the DM indeed faces some discomfort from information. In such a case the tradeoff between emotional costs and physical benefits of information arises. The way in which such trade-off is solved precisely depends on the interplay between the degree of information aversion and the level of uncertainty. For example, when information aversion is low and the cost of an inefficient action is high, anticipatory utility is monotonically increasing in information accuracy. However, if both information aversion and the cost of an inefficient action are low, then two cases can occur: either anticipatory utility is monotonically increasing in information accuracy or it exhibits a maximum for partial information. The latter case arises only if information aversion is low, but not too much (otherwise full information is clearly optimal) and is due to the fact that since uncertainty is small, by choosing imprecise signals the DM can perfectly tune the physical benefits and the emotional costs of information leading to an interior optimum (no corner solutions). In all the other situations, even though anticipatory utility can be nonmonotone in information accuracy, either full information or full ignorance are the preferred choices.

Our work is closely related to and borrows from Kozsegi (2003) who uses a simple version of the PEU model to explain why, as documented by the medical evidence mentioned before, patients sometimes avoid free information on their health status. As in our model, in Kozsegi ignorance is costly in terms of inefficient actions and the DM is information-averse. A first result shows that, when available information is perfect (i.e. the DM decides to be either fully ignorant or fully informed), full information is always preferred to full ignorance if uncertainty is 
limited enough. Then, in a second result Kozsegi introduces the possibility of partial information acquisition (i.e. non fully-informative signals are also available) and illustrates preferences such that anticipatory utility is monotone in information accuracy: the patient either wants full information or remains completely ignorant since partial information is always dominated.

By fully characterizing anticipatory utility we extend and complement Kozsegi's results. In our model, even if uncertainty is limited (as in Kozsegi's first result), still an anomalous behavior can emerge. When information aversion is low (but not too much), the non-monotonicity of anticipatory utility implies that, with small uncertainty, (i) full-ignorance can dominate fullinformation; (ii) although full-information can dominate full-ignorance, partial information dominates full-information. By showing that partial information accuracy can indeed maximize anticipatory utility in some cases, our paper indicates that the PEU model provides an appropriate framework that delivers a complete picture of anomalous attitudes towards information. ${ }^{3}$

From a policy perspective our results show that the disclosure of partial information can be beneficial to anxious DMs. A similar conclusion is reached in two other papers investigating very different set-ups. First, Caplin and Eliaz (2003, page 633) concerning the AIDS test write: "There are strong health-based incentives to test for AIDS, but fear may override these incentives. Our resolution of the problem is to decrease the informativeness of a bad test result, mitigating the fear of bad news, and thereby allowing the health-based incentives to reassert their primacy". Second, Yariv (2005) constructs a multi-period choice model to investigate Cognitive Consistency, that is individuals' natural inclination to interpret new evidence in ways that confirm their pre-existing beliefs. Given the specific aim of his analysis, Yariv assumes that in each period the DM receives a signal and then directly chooses his belief (instead of updating it). An incorrect belief may be advantageous because it is a 'desirable' belief and thus increases anticipatory utility. However the DM is constrained to choose an action which is optimal w.r.t. his belief, such that holding an incorrect belief is costly in terms of physical utility. As a result of such trade-off, the author finds that agents may sometimes prefer less accurate signals to more

\footnotetext{
${ }^{3}$ Eliaz and Spiegler (2006) is also related to our analysis. These authors investigate a general model where the DM's beliefs enter utility (the model of Expected Utility from Beliefs). With such a model they analyze the DM's attitude towards information and information sources. In particular, the authors aim to verify whether the model of Expected Utility from Beliefs is able to explain those situations where the attitude towards information is affected by the DMs' priors (for example, the case of a patient preferring more accurate medical tests when he is relatively certain of being healthy, yet avoiding such tests when he is relatively certain of being ill). The main difference between Eliaz and Spiegler's and C-L's approach is that the latter examine the DM's behavior for a fixed prior while Eliaz and Spiegler account for attitudes to information that vary with the DM's prior.
} 
accurate ones. $^{4}$

The paper is organized as follows. In Section 2 we present the model set-up. In Section 3 we discuss the DM's decision process and derive anticipatory utility as a function of information accuracy. In section 4, we show conditions such that the emotional cost of information is positive, we characterize anticipatory utility and discuss anomalous attitudes towards information. Section 5 concludes. The appendix completes our analysis and provides some numerical simulations.

\section{The model}

Following the methodology of Caplin and Leahy (2001, 2004), the DM's utility is a function of physical outcomes and belief-based emotions, with anticipatory emotions responding to information. There are two periods, 1 and 2, and total utility is the sum of future utility from physical outcomes, and current anticipatory emotions, which depend on rationally formed beliefs about the exact same outcomes. ${ }^{5}$

The DM's physical utility in period 2 is $h\left(w_{i}, a\right)$, where $w_{i}=\left\{w_{1}, w_{2}\right\}$ is the physical outcome realized in period 1 , with $w_{i} \in \Re$, and $a$ is an action. Importantly and as in the case of the motivating examples before, even if the physical outcome is realized in period $1, w_{i}$ has physical effects only in period 2 . In the same way the action $a \in \Re$ is taken in period 1 but it only affects utility in period $2 .^{6}$

We assume that $w_{1}$ is the preferred outcome such that $w_{1}>w_{2}$. Moreover, each outcome has

\footnotetext{
${ }^{4}$ The issue of information gathering by emotional DMs is also related to the recent literature on Selective Attention. This literature develops the idea that individuals consciously decide what information to expose themselves to and what information to avoid. In particular, while people search selectively for information that conveys good news or that reinforces their pre-existing beliefs, they avoid information that conveys bad news or conflicts with prior beliefs. The idea of self-manipulation of beliefs to increase one's utility is particularly natural in the field of finance: recently Karlsson, Loewenstain and Seppi (2005) analyzed investors' selective exposure to information on financial markets in a model where individuals condition their information learning decisions on imperfect prior information that can be positive or negative. They show that investors who observed (imperfect) bad news exhibit the so-called 'ostrich effect'.

${ }^{5}$ This represents a simple version of the PEU model described in C-L (2001). In our model, as in C-L (2004) and in $\mathrm{K}$, the DM experiences only emotional utility in the first period and not both emotional and physical utility as in the seminal model.

${ }^{6}$ In our motivating examples the patient's action is represented by all preventative behavior adopted or treatment undertaken, whereas in the case of the investor, the action can be interpreted as every possible change in his portfolio composition. The action for the suspicious partner could be the appropriate attitude to take with his/her partner.
} 
the same probability $p_{i}, i=1,2$ of occurring, $p_{1}=p_{2}=1 / 2$, and the DM's priors are correct.

By observing a signal the DM obtains information on the outcome realized. A signal is a random variable which can take two values, $s_{1}$ and $s_{2}$. A signal is characterized by a pair of conditional probabilities $\left(q_{1}, q_{2}\right)$, where $q_{i} \in\left[\frac{1}{2}, 1\right], i=1,2$, is the probability of observing the realization $s_{i}$ conditional on the outcome being $w_{i}: q_{i}=\operatorname{prob}\left(s_{i} \mid w_{i}\right)$. For the sake of tractability we also assume that $q_{1}=q_{2}=q$, that is, the signal is symmetric.

Since in the first period the DM derives utility from the anticipation of period-2 physical outcome, anticipatory utility depends on expected physical utility in period 2 conditional on the DM's beliefs in period 1. In turn, the DM's beliefs depend on the signal observed:

$$
\text { emotional utility }=u\left(E\left[h(w, a) \mid s_{i}\right]\right)
$$

The function $u(\cdot)$ is increasing in the expectation of physical utility $E[h(w, a)]$. The shape of $u(\cdot)$ determines the DM's preferences for information. When $u(\cdot)$ is concave, the DM is averse to information and he dislikes bad news more than he likes good news. If $u(\cdot)$ is convex, on the contrary, the DM is 'information-loving'. Finally, when $u(\cdot)$ is linear the DM is 'informationneutral'.

Utility in period 2 corresponds to physical utility $h\left(w_{i}, a\right)$. To calculate the total DM utility from the perspective of period 1, we add to emotional utility (2.1) the expectation of period-2 physical utility: $E\left[h(w, a) \mid s_{i}\right]$.

As is shown in appendix 6.5 , by considering total utility we obtain results that are qualitatively equivalent to those we obtain by restricting ourselves to emotional utility. For this reason, in the rest of the paper we focus on anticipatory utility $(2.1){ }^{7}$

Let's assume that physical utility is $h\left(w_{i}, a\right)=w_{i}-\left(w_{i}-a\right)^{2}$. Thus, given the outcome $w_{i}, w_{i}$ also corresponds to the maximum level of physical utility that can be achieved if the appropriate action $\left(a=w_{i}\right)$ is chosen. If the taken action $a$ is not appropriate, the DM will be worse off: the loss function $\left(w_{i}-a\right)^{2}$ measures the damage resulting from inaccurate actions.

The DM chooses the precision of the signal $q$. The decision to become completely informed is equivalent to the choice of $q=1$, the fully informative signal, whereas the decision to remain ignorant is equivalent to the choice of $q=\frac{1}{2}$, the uninformative signal. The decision to become partially informed corresponds to the choice of an intermediate value for $q$.

\footnotetext{
${ }^{7}$ This approach is also used in $\mathrm{K}$, so that our results will be directly comparable with those of $\mathrm{K}$.
} 
The DM's choice of the precision of the signals, given the prior $p=1 / 2$, is rational. When choosing $q$, the DM anticipates both that he will update his beliefs upon observing the signal's realization and that he will choose the action $a$ according to such beliefs. Information is completely costless.

To summarize, the DM maximizes his emotional utility (2.1). First he chooses the signal precision $q$ and observes the realization of the signal, then he updates his priors according to Bayes' rule, and finally he chooses the optimal action $a$ given updated beliefs. All the actions take place in period 1 .

We assume that the function $u(\cdot)$ is concave. In fact, as will be clear in Section 4, no interesting trade-off exists between the physical benefits and emotional costs of information for DMs who are not information-averse.

Let's calculate posterior beliefs in our simple model. Given priors $p=\frac{1}{2}$ and conditional probabilities $q=\operatorname{prob}\left(s_{i} \mid w_{i}\right), i=1,2$, the probabilities of the two signals and posterior beliefs are indicated in the following table:

\begin{tabular}{|c|c|c|}
\hline$s_{i}$ & $\operatorname{prob}\left(s_{i}\right)$ & $\operatorname{prob}\left(w_{1} \mid s_{i}\right)$ \\
\hline$s_{1}$ & $1 / 2$ & $q$ \\
\hline$s_{2}$ & $1 / 2$ & $1-q$ \\
\hline
\end{tabular}

(Table 1: posterior beliefs.)

We call $z_{i}$ the posterior probability of the preferred outcome $w_{1}$, given that the signal $s_{i}$ has been observed: $z_{i} \equiv \operatorname{prob}\left(w_{1} \mid s_{i}\right)$. Note that, in this simple setting, conditional probability $q$ corresponds to the updated belief that the true state is $w_{1}$, given the signal $s_{1}$. In other words, in our model by choosing the precision of the signal $q$, the DM directly chooses the posterior belief associated with each signal.

\section{The DM's problem}

We solve the DM's problem by backward induction.

- Choice of $a$. At the end of period 1 the DM updates his beliefs and chooses the action $a$ given the signal observed. The optimal action is:

$$
a_{i}^{*}\left(q ; w_{1}, w_{2} \mid s_{i}\right)=\arg \max _{a} u\left(E\left[w-(w-a)^{2} \mid s_{i}\right]\right)
$$


Note that if the signal is fully or partially informative, two different optimal actions exist, whereas if the signal is uninformative, the two optimal actions are the same: $a_{i}^{*}\left(\frac{1}{2} ; w_{1}, w_{2} \mid s_{i}\right)$ $=a^{*}\left(\frac{1}{2} ; w_{1}, w_{2}\right)$.

- Choice of $q$. At the beginning of period 1 the DM chooses the precision of the signal by maximizing anticipatory utility:

$$
\begin{aligned}
U\left(q ; w_{1}, w_{2}\right)= & \frac{1}{2} u\left(E\left[w-\left(w-a_{1}^{*}\left(q ; w_{1}, w_{2} \mid s_{1}\right)\right)^{2} \mid s_{1}\right]\right)+ \\
& \frac{1}{2} u\left(E\left[w-\left(w-a_{2}^{*}\left(q ; w_{1}, w_{2} \mid s_{2}\right)\right)^{2} \mid s_{2}\right]\right)
\end{aligned}
$$

The previous expression represents anticipatory utility from an ex-ante perspective: when choosing information accuracy, the DM anticipates that, if the signal $s_{i}$ is observed, the optimal action will be $a_{i}^{*}$ with expected physical utility $E\left[w-\left(w-a_{i}^{*}\right)^{2} \mid s_{i}\right]$. Thus, each of the two terms in (3.1) measures anticipatory utility deriving from the observation of a specific signal. The two terms are weighed by the probability $\frac{1}{2}$ that each signal is observed.

\subsection{Deriving anticipatory utility}

Since $z_{i}$ is the posterior probability of the outcome $w_{1}$ when the signal $s_{i}$ has been observed, expected physical utility becomes:

$$
E\left[w-(w-a)^{2} \mid s_{i}\right]=z_{i}\left[w_{1}-\left(w_{1}-a\right)^{2}\right]+\left(1-z_{i}\right)\left[w_{2}-\left(w_{2}-a\right)^{2}\right]
$$

It is easy to verify that, given the signal observed, the optimal action corresponds to the mean of the two outcomes weighed by the posterior beliefs:

$$
a_{i}^{*}\left(q ; w_{1}, w_{2} \mid s_{i}\right)=z_{i} w_{1}+\left(1-z_{i}\right) w_{2}=E_{z_{i}}\left(w_{i}\right)
$$

Since $q \geq 1-q$ and $w_{1}>w_{2}$, from Table 1 it follows that $w_{1} \geq a_{1}^{*}\left(q ; w_{1}, w_{2} \mid s_{1}\right) \geq a_{2}^{*}\left(q ; w_{1}, w_{2} \mid s_{2}\right)$ $\geq w_{2}$. In fact, signal $s_{1}$ represents 'good news' for the DM because the preferred outcome $w_{1}$ is more likely: $a_{1}^{*}\left(q ; w_{1}, w_{2} \mid s_{1}\right)$ is relatively closer to $w_{1}$. On the contrary, signal $s_{2}$ represents 'bad news' for the DM because the preferred outcome $w_{1}$ is more unlikely: $a_{2}^{*}\left(q ; w_{1}, w_{2} \mid s_{2}\right)$ is relatively closer to $w_{2}$. When the signal is fully informative $a_{1}^{*}\left(1 ; w_{1}, w_{2} \mid s_{1}\right)=w_{1}$ and $a_{2}^{*}\left(1 ; w_{1}, w_{2} \mid s_{2}\right)=$ 
$w_{2}$, whereas when the signal is uninformative the unique optimal action is $a^{*}\left(\frac{1}{2} ; w_{1}, w_{2}\right)=$ $\frac{w_{1}+w_{2}}{2}$. In general, as the signal becomes more informative, the action $a_{i}^{*}\left(q ; w_{1}, w_{2} \mid s_{i}\right)$ becomes more accurate on average and the DM's expected physical loss decreases.

By substituting (3.3) in (3.2) and rearranging we find indirect expected physical utility when signal $s_{i}$ is observed:

$$
f_{i}\left(q ; w_{1}, w_{2}\right) \equiv E\left[w-\left(w-a_{i}^{*}\left(q ; w_{1}, w_{2} \mid s_{i}\right)\right)^{2} \mid s_{i}\right]=E_{z_{i}}\left(w_{i}\right)-q(1-q)\left(w_{1}-w_{2}\right)^{2}
$$

Note that $q(1-q)\left(w_{1}-w_{2}\right)^{2}$ represents the expected physical loss due to an inaccurate action and is decreasing in $q$. Moreover, indirect expected physical utility $f_{i}\left(q ; w_{1}, w_{2}\right), i=1,2$, is convex in $q$. By calculating (3.3) and (3.4) for the two signals $s_{1}$ and $s_{2}$ and substituting them in (3.1), we find anticipatory utility as a function of the precision of the signal $q$ :

$$
\begin{aligned}
U\left(q ; w_{1}, w_{2}\right)= & \frac{1}{2} u\left(q w_{1}+(1-q) w_{2}-q(1-q)\left(w_{1}-w_{2}\right)^{2}\right)+ \\
& \frac{1}{2} u\left((1-q) w_{1}+q w_{2}-q(1-q)\left(w_{1}-w_{2}\right)^{2}\right)
\end{aligned}
$$

The DM's problem we analyze is:

$$
\left\{\begin{array}{l}
\max _{q} U\left(q ; w_{1}, w_{2}\right) \\
\text { s.t. }: q \in\left[\frac{1}{2}, 1\right]
\end{array}\right.
$$

\section{Preferences for information accuracy}

Suppose for a moment that physical utility is simply $w_{i}$ and no action has to be taken; noinformation always dominates information learning in this case. In fact, starting from $q=\frac{1}{2}$ and slightly increasing $q$, the expected outcome conditional on signal $s_{1}\left(E_{z_{1}}(w)=q w_{1}+\right.$ $\left.(1-q) w_{1}\right)$ moves slightly from $E(w) \equiv \frac{1}{2} w_{1}+\frac{1}{2} w_{2}$ towards the preferred outcomes $w_{1}$, whereas the expected outcome conditional on signal $s_{2}\left(E_{z_{2}}(w)=(1-q) w_{1}+q w_{2}\right)$ moves slightly from $E(w)$ towards the outcome $w_{2}$. Since the function $u(\cdot)$ is concave, Jensen's inequality implies that discomfort from information increases with the distance between the two expected outcomes $E_{z_{1}}(w)$ and $E_{z_{2}}(w)$. When the signal is fully informative, discomfort reaches its maximum since, in that case, $E_{z_{1}}(w)=w_{1}$ and $E_{z_{2}}(w)=w_{2}$. Thus: 
Remark 1. When information has no decision-making value, discomfort from information is captured by the following expression and is increasing in information accuracy:

$$
u(E(w))-\left[\frac{1}{2} u\left(E_{z_{1}}(w)\right)+\frac{1}{2} u\left(E_{z_{2}}(w)\right)\right]
$$

Expression (4.1) will be extended to the case where the action is available in the next subsection.

Let's now consider the function $U\left(q ; w_{1}, w_{2}\right)$ in the two extreme cases: the uninformative and the fully informative signal. From (3.5), when the signal is uninformative anticipatory utility is:

$$
U\left(\frac{1}{2} ; w_{1}, w_{2}\right)=u\left(\frac{w_{1}+w_{2}}{2}-\frac{1}{4}\left(w_{1}-w_{2}\right)^{2}\right)
$$

Here there is no information gathering and, therefore, no discomfort from information. However the expected physical loss is maximal. Obviously physical costs are increasing in the difference between the two outcomes $w_{1}$ and $w_{2}$.

On the contrary, when the signal is fully informative anticipatory utility is:

$$
U\left(1 ; w_{1}, w_{2}\right)=\frac{1}{2} u\left(w_{1}\right)+\frac{1}{2} u\left(w_{2}\right)
$$

Here there is no physical loss because the action $a$ is completely accurate. Obviously $U\left(1 ; w_{1}, w_{2}\right)$ is lower (or discomfort from information is higher) the more concave the function $u(\cdot)$.

\subsection{Non-monotonicity of anticipatory utility}

We can now characterize anticipatory utility as a function of information accuracy. Recall that indirect expected physical utility conditional on the signal $s_{i}$ is $f_{i}\left(q ; w_{1}, w_{2}\right) \equiv E\left[w-\left(w-a_{i}^{*}\right)^{2} \mid s_{i}\right]$, $i=1,2$, as expressed in (3.4). Thus, anticipatory utility (3.5) can be rewritten as:

$$
U\left(q ; w_{1}, w_{2}\right)=\frac{1}{2} u\left(f_{1}\left(q ; w_{1}, w_{2}\right)\right)+\frac{1}{2} u\left(f_{2}\left(q ; w_{1}, w_{2}\right)\right)
$$

By deriving with respect to $q$ we find:

$$
\frac{\partial U\left(q ; w_{1}, w_{2}\right)}{\partial q}=\frac{1}{2} u_{1}^{\prime}\left(f_{1}\right) f_{1}^{\prime}\left(q ; w_{1}, w_{2}\right)+\frac{1}{2} u_{2}^{\prime}\left(f_{2}\right) f_{2}^{\prime}\left(q ; w_{1}, w_{2}\right)
$$


where $u_{i}^{\prime}(\cdot)$ and $f_{i}^{\prime}(\cdot), i=1,2$, respectively are the first derivative of $u(\cdot)$ with respect to $f_{i}\left(q ; w_{1}, w_{2}\right)$ and the first derivative of $f_{i}\left(q ; w_{1}, w_{2}\right)$ with respect to $q$. As stated before, $u(\cdot)$ is increasing and concave and $f_{i}\left(q ; w_{1}, w_{2}\right), i=1,2$, is convex in $q$.

Using expression (3.4), the first derivatives of $f_{1}\left(q ; w_{1}, w_{2}\right)$ and $f_{2}\left(q ; w_{1}, w_{2}\right)$ with respect to $q$ respectively are:

$$
\begin{aligned}
& f_{1}^{\prime}\left(q ; w_{1}, w_{2}\right)=\left(w_{1}-w_{2}\right)\left[1+\left(w_{1}-w_{2}\right)(2 q-1)\right] \\
& f_{2}^{\prime}\left(q ; w_{1}, w_{2}\right)=\left(w_{1}-w_{2}\right)\left[-1+\left(w_{1}-w_{2}\right)(2 q-1)\right]
\end{aligned}
$$

Note that, when $q=\frac{1}{2}, f_{i}\left(q ; w_{1}, w_{2}\right)=f\left(\frac{1}{2} ; w_{1}, w_{2}\right)=\frac{w_{1}+w_{2}}{2}-\frac{1}{4}\left(w_{1}-w_{2}\right)^{2}, i=1,2$, and the derivative (4.4) is always equal to zero. This is a consequence of the quadratic loss function we use in DM's physical utility.

Remark 2. For $q=\frac{1}{2}$, the first derivative of $U\left(q ; w_{1}, w_{2}\right)$ is always zero: the uninformative signal corresponds either to a local maximum or to a local minimum.

Whether the uninformative signal corresponds to a local maximum or to a local minimum of anticipatory utility depends on the degree of the DM's aversion to information. This will be analyzed in Lemma 1.

Now we consider the sign of expression (4.4). From (4.5) $f_{1}^{\prime}(\cdot)$ is positive. This implies that the first term in (4.4) is always positive, whereas the second term has the same sign as $f_{2}^{\prime}(\cdot)$. Thus, if $f_{2}(\cdot)$ is increasing in $q$ for $q \in\left[\frac{1}{2}, 1\right]$, anticipatory utility is monotonically increasing in information accuracy and full information gathering is obviously optimal. When, on the contrary, $f_{2}(\cdot)$ is decreasing in $q$, the first and the second term in (4.4) have opposite signs such that the derivative of anticipatory utility can be either positive or negative. This shows that the sign of $f_{2}^{\prime}(\cdot)$ is crucial to assess whether partial or no information gathering are optimal choices.

For an economic intuition, note that an increase in the precision of the signal $q$ always leads to an increase in $f_{1}\left(q ; w_{1}, w_{2}\right)$ since, when the signal $s_{1}$ is observed, (a) the preferred outcome is more likely and (b) the physical loss due to the inaccurate action decreases, whereas an increase in the precision of the signal $q$ makes $f_{2}\left(q ; w_{1}, w_{2}\right)$ either increase or decrease. In fact, when the signal $s_{2}$ is observed, (a) the bad outcome is more likely (this corresponds to the term -1 in (4.6)), (b) the physical loss due to the inaccurate action decreases (this corresponds to the term $+\left(w_{1}-w_{2}\right)(2 q-1)$ in 4.6). If the negative effect of information on $f_{2}(\cdot)$ prevails 
$\left(f_{2}(\cdot)\right.$ decreasing in $\left.q\right)$, starting from $q=\frac{1}{2}$, information gathering shifts $f_{2}(\cdot)$ to the left of $f\left(\frac{1}{2} ; w_{1}, w_{2}\right)=\frac{w_{1}+w_{2}}{2}-\frac{1}{4}\left(w_{1}-w_{2}\right)^{2}$ and $f_{1}(\cdot)$ to its right such that the expected physical utilities $f_{1}(\cdot)$ and $f_{2}(\cdot)$ diverge and some discomfort from information arises. We now define discomfort from information in the case where information has decision-making value: ${ }^{8}$

Remark 3. When information has decision-making value, discomfort from information only arises if $f_{2}(\cdot)$ is decreasing in information accuracy for at least a sub-interval in $\left[\frac{1}{2}, 1\right]$. In such a sub-interval discomfort from information is positive and equal to:

$$
u\left(f\left(\frac{1}{2} ; w_{1}, w_{2}\right)\right)-\left[\frac{1}{2} u\left(f_{1}\left(q ; w_{1}, w_{2}\right)\right)+\frac{1}{2} u\left(f_{2}\left(q ; w_{1}, w_{2}\right)\right)\right]
$$

Note that, when some discomfort from information arises, the DM faces a real trade-off between the emotional costs and the physical benefits of information. Importantly, only in such a case anomalous attitudes towards information can occur.

In the next remark we show how the distance between the two outcomes $w_{1}-w_{2}$ affects the derivative of $f_{2}\left(q ; w_{1}, w_{2}\right)$. Starting from $q=\frac{1}{2}$ and slightly increasing information accuracy, first the negative effect of information on $f_{2}\left(q ; w_{1}, w_{2}\right)$ prevails over the positive effect independently of $w_{1}-w_{2}$. Then, as information accuracy increases, the derivative of $f_{2}\left(q ; w_{1}, w_{2}\right)$ strictly depends on the distance between the two outcomes. In particular, as $q$ increases, the negative effect due to beliefs getting worse still dominates for $w_{1}-w_{2}<1$, whereas the positive effect due to the more efficient action prevails for $w_{1}-w_{2} \geq 1$. Thus, when $w_{1}-w_{2}<1, f_{2}\left(q ; w_{1}, w_{2}\right)$ is always decreasing in $q$ for $q \in\left[\frac{1}{2}, 1\right]$.

Remark 4. (i) When $w_{1}-w_{2}<1, f_{2}^{\prime}\left(q ; w_{1}, w_{2}\right)<0$ for $q \in\left[\frac{1}{2}, 1\right]$. (ii) When $w_{1}-w_{2} \geq 1$, $f_{2}^{\prime}(\cdot) \geq 0$ for $q=1$ such that $\left.\frac{\partial U\left(q ; w_{1}, w_{2}\right)}{\partial q}\right|_{q=1}>0$. (iii) When $w_{1}-w_{2} \geq 1, f_{2}\left(q ; w_{1}, w_{2}\right)$ is first decreasing and then increasing in $q$ in the interval $\left[\frac{1}{2}, 1\right]$. In particular, the larger $w_{1}-w_{2}$, the lower the subinterval of $\left[\frac{1}{2}, 1\right]$ in which $f_{2}(\cdot)$ is decreasing in $q$.

$\mathbf{P}$ roof. See the appendix 6.1

The previous remark shows that, when the cost of an inefficient action is low $\left(w_{1}-w_{2}<1\right)$, discomfort from information is always positive and the DM faces the trade-off between the

\footnotetext{
${ }^{8}$ Note that discomfort from information acquisition is always positive and increasing in information accuracy when no action is available. Whereas, when information has decision-making value, it occurs only if unfavorable updated beliefs due to bad news have a stronger impact on anticipatory utility than better choices. Moreover, in that case, discomfort from information is not necessarily increasing in information accuracy.
} 
emotional costs and physical benefits of information whatever the value of information accuracy. When, on the contrary, the cost of an inefficient action is high $\left(w_{1}-w_{2} \geq 1\right)$, discomfort from information is positive for low values of information accuracy and disappears for high value of $q$. Put differently, high uncertainty implies that better actions always offset the effect of a bad signal on beliefs for sufficiently high information accuracy. Thus, in such a case, information is always beneficial when $q$ approaches one.

Let's now consider the second derivative of the function $U\left(q ; w_{1}, w_{2}\right)$ :

$$
\frac{\partial^{2} U\left(q ; w_{1}, w_{2}\right)}{\partial q^{2}}=\frac{1}{2} u_{1}^{\prime \prime}\left(f_{1}\right)\left(f_{1}^{\prime}(\cdot)\right)^{2}+\frac{1}{2} u_{1}^{\prime}\left(f_{1}\right) f_{1}^{\prime \prime}(\cdot)+\frac{1}{2} u_{2}^{\prime \prime}\left(f_{2}\right)\left(f_{2}^{\prime}(\cdot)\right)^{2}+\frac{1}{2} u_{2}^{\prime}\left(f_{2}\right) f_{2}^{\prime \prime}(\cdot)
$$

where $u_{i}^{\prime \prime}(\cdot)$ and $f_{i}^{\prime \prime}(\cdot), i=1,2$, respectively are the second derivative of $u(\cdot)$ with respect to $f_{i}\left(q ; w_{1}, w_{2}\right)$ and the second derivative of $f_{i}\left(q ; w_{1}, w_{2}\right)$ with respect to $q$. Note that, since $u(\cdot)$ is increasing in expected physical utility and expected physical utility is convex w.r.t. $q$, the second term and the last one of (4.7) are both positive whereas, given our assumption on the sign of $u_{i}^{\prime \prime}(\cdot)$, the first and third terms are both negative (whatever the sign of $\left.f_{2}^{\prime}(\cdot)\right){ }^{9}$

We now look for conditions such that the uninformative signal corresponds either to a local maximum or to a local minimum of anticipatory utility. To do so we must define the DM's relative aversion to information adapting the concept of relative risk aversion to anticipatory utility:

Definition 1. Relative aversion to information corresponds to $R_{u}(f)=-f\left(q ; w_{1}, w_{2}\right) \frac{u^{\prime \prime}\left(f\left(q ; w_{1}, w_{2}\right)\right)}{u^{\prime}\left(f\left(q ; w_{1}, w_{2}\right)\right)}$.

Lemma 1. (i) The uninformative signal is a local minimum if

$$
R_{u}\left(f_{\frac{1}{2}}\right)<w_{1}+w_{2}-\frac{1}{2}\left(w_{1}-w_{2}\right)^{2} .
$$

Inequality (4.8) has economic meaning if its r.h.s. is positive, that is for $w_{1} \in\left(w_{2}+1-\sqrt{4 w_{2}+1}\right.$, $\left.w_{2}+1+\sqrt{4 w_{2}+1}\right)$.

(ii) The uninformative signal is a local maximum if the opposite of (4.8) holds.

$\mathbf{P}$ roof. See the appendix 6.2 .

\footnotetext{
${ }^{9}$ When the function $u(\cdot)$ is convex or linear, expression (4.7) is always positive. This means that anticipatory utility is monotonically increasing in $q$ and exhibits a global minimum for $q=\frac{1}{2}$ : as we expected, an informationloving or information-neutral DM will always choose full information gathering.
} 
The degree of the DM's relative information aversion is crucial for the non-monotonicity of anticipatory utility, as we will see.

Now we look for sufficient conditions for partial information gathering to be the DM's preferred choice. In Appendix 6.3 we show that anticipatory utility is decreasing in the signal precision for the fully informative signal if inequality (6.2) holds. Two necessary conditions are: $w_{1}-w_{2}<1$ and the function $u(\cdot)$ concave enough. In other words, anticipatory utility can be decreasing in the signal precision for $q=1$ only if the inaccurate action is not too costly and if aversion to information is sufficiently high. Inequalities (4.8) and (6.2) (the latter in the appendix) represent sufficient conditions such that problem P1 exhibits an internal solution and anticipatory utility is as in figures 1 and 2 :

Remark 5. If the uninformative signal is a minimum of anticipatory utility and anticipatory utility is decreasing in the signal precision for the fully informative signal, that is if inequalities (4.8) and (6.2) simultaneously hold, a partially informative signal is the DM's optimal choice.

\section{Insert figure 1 and 2 about here}

Note that condition (4.8) requires that $u(\cdot)$ is not too concave while a necessary condition for (6.2) is that $u(\cdot)$ must be concave enough. That is, as we expect, an internal solution is possible when information aversion is neither too high nor too low. In fact, in such a case, even if the uninformative signal is a minimum of anticipatory utility, for sufficiently high information accuracy full information is dominated by partial information (see the discussion of case (a) after Proposition 1). In Appendix 6.4 our simulations show that, with the power function, inequalities (4.8) and (6.2) can both be met.

We are now ready to characterize anticipatory utility and to explain why it can be nonmonotone in the signal precision. The shape of $U\left(q ; w_{1}, w_{2}\right)$ depends both on the DM's information aversion, captured by the concavity of the function $u(\cdot)$, and on the costs of the inaccurate action, captured by the distance between the two possible outcomes $w_{1}$ and $w_{2}$.

Before stating our proposition we recall that $f_{2}^{\prime}(\cdot)<0$ is a necessary condition for anticipatory utility to be decreasing in information accuracy (see Remark 3). Moreover, the sign of $f_{2}^{\prime}(\cdot)$ depends on the distance between $w_{1}$ and $w_{2}$ (see Remark 4).

Proposition 1. The shape of anticipatory utility depends on the interaction between the degree 
of the DM's information aversion and the physical costs due to an inaccurate action. According to the model parameters the DM's optimal choice can be the fully informative signal, the uninformative one, or a partially informative signal as summarized by the table below.

\begin{tabular}{|l|c|c|}
\hline & $q=\frac{1}{2}$ is a (local) minimum & $q=\frac{1}{2}$ is a (local) maximum \\
\hline$w_{1}-w_{2}<1$ & $\begin{array}{c}\text { case (a) } \\
\text { either partial or full information }\end{array}$ & $\begin{array}{c}\text { cather no or full information } \\
\text { case (d) }\end{array}$ \\
\hline$w_{1}-w_{2} \geq 1$ & $\begin{array}{c}\text { case } \text { call information } \\
\text { full information }\end{array}$ \\
\hline
\end{tabular}

(Table 2: optimal choices depending on the parameters.)

In the following the four possible cases are illustrated and discussed.

- Case (a): $q=\frac{1}{2}$ corresponds to a (local) minimum and $w_{1}-w_{2}<1$. Expected physical utility $f_{2}(\cdot)$ is decreasing in $q$ for $q \in\left[\frac{1}{2}, 1\right]$; this implies that the DM experiments discomfort from information whatever the value of information accuracy. Aversion to information is (rather) low as well as the damage due to ignorance: the trade-off between the emotional costs and physical benefits of information arises for all values of information accuracy. Since the uninformative signal is a local minimum, anticipatory utility is necessarily increasing in $q$ near $q=\frac{1}{2}$. This means that the physical benefits dominate the emotional costs of information for low values of information accuracy. However, as information accuracy increases, two sub-cases are possible: either the positive term in (4.4) dominates or the negative one dominates. When the positive term $u_{1}^{\prime}\left(f_{1}\right) f_{1}^{\prime}(\cdot)$ prevails, then $q=\frac{1}{2}$ corresponds to a global minimum and full information is the optimal choice since anticipatory utility is monotonically increasing in $q$. The physical benefits always dominate the emotional costs of information in this sub-case. On the contrary, when the negative term $u_{2}^{\prime}\left(f_{2}\right) f_{2}^{\prime}(\cdot)$ prevails, then anticipatory utility is decreasing in information accuracy for values of $q$ close to 1: for high values of information accuracy the emotional costs dominate the physical benefits of information. Here partial information gathering is the optimal choice. According to Remark 5 this sub-case occurs if both inequalities 4.8 and 6.2 are verified. Interestingly, due to the non-monotonicity of anticipatory utility, in this sub-case either the fully informative signal dominates the uninformative one, or the opposite occurs (see figures 1 and 2). 
- Case (b): $q=\frac{1}{2}$ corresponds to a (local) minimum and $w_{1}-w_{2} \geq 1$. Expected physical utility $f_{2}(\cdot)$ is decreasing in $q$ only for low values of information accuracy; this implies that the DM experiments discomfort from information only close to $q=\frac{1}{2}$. Moreover, as before, since the uninformative signal is a local minimum, anticipatory utility is necessarily increasing in $q$ near $q=\frac{1}{2}$. Thus, the physical benefits dominate the emotional costs of information for low values of information accuracy. Since $f_{2}(\cdot)$ is increasing in $q$ for high values of $q$, anticipatory utility is also necessarily increasing in information accuracy for high information accuracy. We infer that anticipatory utility is monotonically increasing in $q$ in the interval $\left[\frac{1}{2}, 1\right]$ : the uninformative signal corresponds to a global minimum and full information gathering is the optimal choice.

- Case (c): $q=\frac{1}{2}$ corresponds to a (local) maximum and $w_{1}-w_{2}<1$. Expected physical utility $f_{2}(\cdot)$ is decreasing in $q$ in the whole interval $\left[\frac{1}{2}, 1\right]$; this implies that the DM experiments discomfort from information whatever the value of information accuracy and the trade-off between the emotional costs and physical benefits of information always arises. Since the fully uninformative signal is a local maximum, anticipatory utility is necessarily decreasing in $q$ near $q=\frac{1}{2}$. This means that the emotional costs dominate the physical benefits of information close to $q=\frac{1}{2}$. As in case (a) before, when information accuracy increases two sub-cases are possible: either the positive term in (4.4) dominates or the negative one dominates. When the negative term $u_{2}^{\prime}\left(f_{2}\right) f_{2}^{\prime}(\cdot)$ prevails, then anticipatory utility is monotonically decreasing in information accuracy for $q \in\left[\frac{1}{2}, 1\right]$; in such a subcase the emotional costs always dominate the physical benefits of information and $q=\frac{1}{2}$ corresponds to a global maximum. On the contrary, when the positive term $u_{1}^{\prime}\left(f_{1}\right) f_{1}^{\prime}(\cdot)$ prevails, anticipatory utility becomes increasing in information accuracy for values of $q$ near to 1 . In this sub-case the physical benefits dominate the emotional costs of information for high values of information accuracy and anticipatory utility exhibits a global minimum for partial information gathering. The preferred choice can be either the uninformative signal or the fully informative one. Note that this latter result is quite counterintutive: full information gathering can be the preferred choice even though the cost of the inaccurate action is low and aversion to information is high. As in the second sub-case in (a), this depends on the non-monotonicity of anticipatory utility which here necessarily implies a corner solution. 
- Case (d): $q=\frac{1}{2}$ corresponds to a (local) maximum and $w_{1}-w_{2} \geq 1$. Since $w_{1}-w_{2} \geq 1$, expected physical utility $f_{2}(\cdot)$ is first decreasing and then increasing in $q$ in the interval $\left[\frac{1}{2}, 1\right]$. Thus, discomfort from information arises when information accuracy is low but disappears when information accuracy is high. Given that the fully uninformative signal is a local maximum, anticipatory utility is decreasing in $q$ near $q=\frac{1}{2}$. This means that the emotional costs dominate the physical benefits of information close to $q=\frac{1}{2}$. However, as the signal becomes more informative, anticipatory utility necessarily becomes increasing in information accuracy. As a consequence anticipatory utility exhibits a global minimum for partial information gathering. The preferred choice can be again either the fully informative signal or the uninformative one. ${ }^{10}$

Details concerning simulations of all the possible cases are provided in Appendix 6.4.

Before concluding this section we illustrate how our results complete and extend those of Kozsegi (2003). Using a linear loss function he shows that (i) the DM always prefers full information to full ignorance if uncertainty is limited, i.e. if news is sufficiently 'small' and does not modify the DM's beliefs very far; (ii) anticipatory utility is monotonically increasing (or partial information is never optimal) when absolute aversion to information is constant. With respect to point (i) we show that, when the loss function is quadratic, no-information can dominate full information even though uncertainty is small (see the first sub-case in (a) above). With a linear loss function this cannot be the case since, even when uncertainty is close to zero, linearity imposes a strictly positive (marginal) benefit from accurate actions (or a strictly positive marginal cost from ignorance). This implies that, for sufficiently small uncertainty, discomfort from information becomes irrelevant whereas the benefit from full information does not, such that full information necessarily dominates full ignorance. ${ }^{11}$ Again concerning point (i), since in our model anticipatory utility can be non-monotone when uncertainty is small, even though full information can dominate no-information, partial information can dominate both of

\footnotetext{
${ }^{10}$ Note that, when $q=\frac{1}{2}$ is a maximum, whatever the distance between the two outcomes, the preferred signal is either the fully informative or the uninformative one (see Table 1). However, when $w_{1}-w_{2} \geq 1$, anticipatory utility is always first decreasing and then increasing in information accuracy. On the contrary, when $w_{1}-w_{2}<1$, anticipatory utility can be either first decreasing and then increasing or monotonically decreasing in information accuracy. The latter sub-case occurs when the emotional costs of information always dominate its physical benefits.

${ }^{11}$ In addition to being continuously differentiable, a quadratic loss function differs from a linear one (i.e. $\left.-k\left|w_{i}-a\right|\right)$ in that the cost of ignorance due to imprecise decisions becomes very small and negligible when uncertainty tends to vanish. We believe that both loss functions may then adequately represent different real world environments and are equally interesting.
} 
them. With respect to point (ii) we find general conditions based on the level of uncertainty and on the DM's relative aversion to information such that anticipatory utility is monotonically increasing in information accuracy (see the first sub-case in (a) and case (b)). With a quadratic loss function relative risk aversion is relevant since it determines whether the uninformative signal is a maximum or a minimum of anticipatory utility, which is crucial for non-monotonicity.

\section{Conclusion}

In this paper we have addressed the issue of acquisition of information by an emotional DM, that is an agent who fears bad future outcomes. Using a simple and treatable version of the Psychological Expected Utility model, we showed that the DM's approach towards information gathering depends on the interplay between two key elements: the level of information aversion and the expected damage due to an inaccurate action (which in turn depends on the level of uncertainty). Anticipatory utility can be non-monotone in information accuracy and, depending on the parameters, full information, no information or partial information accuracy can be the preferred choice. More generally, we showed that anticipatory feelings may really lead to less information gathering than one would expect from fully rational DMs.

While well-known empirical evidence exists concerning complete information-avoidance behaviors as for patients and information provided by medical tests (see the papers mentioned in the introduction), instances in which one can completely ignore or avoid signals are relatively rare in other real life situations. Even though, in our daily experience, we observe that partial information gathering is more plausible and frequent than complete information avoidance, empirical evidence for partial information-avoidance behaviors is not easy to obtain. This is why it may be expedient to look for experimental evidence in the lab, instead. We leave that for future research.

A recent literature analyzed information transmission in the agency relationship when the principal experiences anticipatory feelings and a benevolent, privately informed agent takes into account those feelings (see Caplin and Leahy 2004 and Kozsegi 2006). In Kozsegi (2006) the agent is ready to lie if this makes the principal feel better. The principal correctly anticipates this and, as a consequence, credibility problems arise (as sometimes occurs in the patient-physician relationship ${ }^{12}$ ). Information acquisition by the principal from sources other than the agent can

\footnotetext{
${ }^{12}$ For a survey on the important change in the analysis of the patient-physician relationship recently introduced
} 
be introduced into the picture to investigate the role played by endogenous information gathering in principal-agent communication. We believe that our simple framework could provide a useful tool for addressing this issue.

\section{Appendix}

\subsection{Proof of Remark 4}

(i) From (4.6) it is easy to check that, when $w_{1}-w_{2}<1, f_{2}^{\prime}(\cdot)$ is negative for $q \in\left[\frac{1}{2}, 1\right]$. (ii) This is obvious from (4.6) and (4.4). (iii) When $w_{1}-w_{2} \geq 1, f_{2}^{\prime}(\cdot)<0$ for $q=\frac{1}{2}$ and $f_{2}^{\prime}(\cdot)>0$ for $q=1$. Thus, starting from $q=\frac{1}{2}, f_{2}(\cdot)$ is first decreasing and then increasing in $q$. From (4.6) it is easy to see that the higher $w_{1}-w_{2}$, the shorter the subinterval of $\left[\frac{1}{2}, 1\right]$ where $f_{2}(\cdot)$ is decreasing in $q$.

\subsection{Proof of Lemma 1}

(i) When $q=\frac{1}{2}$, it is easy to see that:

$$
\left.\frac{\partial^{2} U\left(q ; w_{1}, w_{2}\right)}{\partial q^{2}}\right|_{q=\frac{1}{2}}=2 u^{\prime}\left(f\left(\frac{1}{2} ; w_{1}, w_{2}\right)\right)+u^{\prime \prime}\left(f\left(\frac{1}{2} ; w_{1}, w_{2}\right)\right)
$$

where $u_{1}^{\prime}(\cdot)=u_{2}^{\prime}(\cdot)=u^{\prime}(\cdot)$, thus:

$$
\left.\frac{\partial^{2} U\left(q ; w_{1}, w_{2}\right)}{\partial q^{2}}\right|_{q=\frac{1}{2}}>0 \Leftrightarrow R_{u}(f)<2 f\left(\frac{1}{2} ; w_{1}, w_{2}\right) .
$$

(ii) This corresponds to the case where $\left.\frac{\partial^{2} U\left(q ; w_{1}, w_{2}\right)}{\partial q^{2}}\right|_{q=\frac{1}{2}}<0$.

\subsection{Anticipatory utility decreasing in the signal precision for $q=1$.}

Remark 6. Anticipatory utility is decreasing in the signal precision for $q=1$ if and only if:

$$
\frac{u^{\prime}\left(w_{1}\right)}{u^{\prime}\left(w_{2}\right)}<\frac{1-\left(w_{1}-w_{2}\right)}{1+\left(w_{1}-w_{2}\right)}
$$

Two necessary conditions are $w_{1}-w_{2}<1$ and $u(\cdot)$ concave enough.

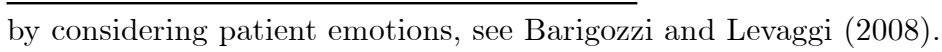


$\mathbf{P}$ roof. When $q=1$, it is easy to see that:

$$
\left.\frac{\partial U\left(q ; w_{1}, w_{2}\right)}{\partial q}\right|_{q=1}=\frac{1}{2}\left(w_{1}-w_{2}+\left(w_{1}-w_{2}\right)^{2}\right) u^{\prime}\left(w_{1}\right)+\frac{1}{2}\left(-\left(w_{1}-w_{2}\right)+\left(w_{1}-w_{2}\right)^{2}\right) u^{\prime}\left(w_{2}\right)
$$

Thus, for $q=1$, anticipatory utility is decreasing in the signal precision if:

$$
\left(1+\left(w_{1}-w_{2}\right)\right) u^{\prime}\left(w_{1}\right)-\left(1-\left(w_{1}-w_{2}\right)\right) u^{\prime}\left(w_{2}\right)<0
$$

From (6.3) condition (6.2) can be immediately derived. Moreover (6.3) can be verified only if $w_{1}-w_{2}<1$ and it implies that $u(\cdot)$ must be concave enough. In fact, inequality (6.2) is verified only if $u_{1}^{\prime}\left(w_{1}\right)$ is sufficiently lower than $u_{2}^{\prime}\left(w_{2}\right)$.

\subsection{Simulations}

Let's consider the power function $u(x)=\frac{x^{1-\gamma}}{1-\gamma}$, with $\gamma$ re-interpreted as the parameter of constant relative aversion to information. ${ }^{13}$ In this paragraph we provide examples of all the possible results described in Table 2 .

- Case (a) Let's start with the more interesting sub-case: a preference for the partial informative signal. According to Remark 5, let's take $\gamma<2 f\left(\frac{1}{2} ; w_{1}, w_{2}\right)$. The uninformative signal is a minimum for $w_{1} \in\left[w_{2}+1-\sqrt{4 w_{2}+1-2 \gamma}, w_{2}+1+\sqrt{4 w_{2}+1-2 \gamma}\right]$ and $\gamma<\frac{1}{2}+2 w_{2}$. Moreover, for $w_{1}-w_{2}<1$ the derivative of anticipatory utility in $q=1$ is negative. ${ }^{14}$ Figures 1 and 2 show anticipatory utility in the interval $q \in\left[\frac{1}{2}, 1\right]$ when $u(\cdot)$ is the power function and $w_{1}=.55, w_{2}=.023$. It can be seen that for $\gamma=.42$ (figure 1 ) the uninformative signal dominates the fully informative one, whereas for $\gamma=.4$ (figure 2) the opposite holds. According to intuition, all else being equal, the lower the relative aversion to information, the closer to 1 the optimal precision of the signal. In Appendix 6.5 we present the case of total utility (anticipatory utility plus expected physical utility): an interior solution still exists and it is compatible with higher values of information aversion.

\footnotetext{
${ }^{13}$ To the best of our knowledge, no empirical parameter estimates of individual relative information aversion are available. The estimates of relative risk aversion vary considerably, but values in the $0.5-3$ interval are often referred to.

${ }^{14}$ Our simulations show that $w_{2}$ must be sufficiently close to zero: when this is the case the power function is concave enough for inequality (6.2) to be satisfied.
} 
The second sub-case arises when $q=\frac{1}{2}$ corresponds to a global minimum and full information is the optimal choice. This occurs, for example, in the case $\gamma=.3, \omega_{1}=.5$ and $\omega_{2}=.2$.

- Case (b) The uninformative signal corresponds to a global minimum and full information gathering is the optimal choice. This occurs, for example, when $\gamma=.5, \omega_{1}=2.5$ and $\omega_{2}=.5$.

- Case (c) In the first sub-case $q=\frac{1}{2}$ is a global maximum and no information is the optimal choice. This occurs, for example, when $\gamma=.7, \omega_{1}=.45$ and $\omega_{2}=.2$. In the second subcase anticipatory utility is first decreasing and then increasing in information accuracy and either full information or no information are the preferred choices. When $\gamma=1.2, \omega_{1}=1$, $\omega_{2}=.3$ full information dominates no information whereas when $\gamma=1.2, \omega_{1}=.99$, $\omega_{2}=.2$, the opposite occurs.

- Case (d) Anticipatory utility exhibits a global minimum for partial information gathering. The preferred choice can be either the fully informative signal or the uninformative one. When $\gamma=1.1, \omega_{1}=1.3, \omega_{2}=.05$ no information dominates full information whereas when $\gamma=.8, \omega_{1}=1.3, \omega_{2}=.05$ the opposite occurs.

\subsection{The case of total utility}

As we mentioned before, using anticipatory utility only instead of total utility (consisting of anticipatory utility plus expected physical utility) as the DM's objective involves no loss of generality. To show that, in this paragraph we obtain sufficient conditions such that partial information is the preferred choice (as in Remark 5) when the DM's well-being is measured by total utility. Total utility is ${ }^{15}$ :

$$
\begin{aligned}
\hat{U}\left(q ; w_{1}, w_{2}\right)= & \frac{1}{2} u\left(q w_{1}+(1-q) w_{2}-q(1-q)\left(w_{1}-w_{2}\right)^{2}\right)+ \\
& \frac{1}{2} u\left((1-q) w_{1}+q w_{2}-q(1-q)\left(w_{1}-w_{2}\right)^{2}\right)+ \\
& \frac{1}{2}\left(w_{1}-w_{2}\right)-q(1-q)\left(w_{1}-w_{2}\right)^{2}
\end{aligned}
$$

\footnotetext{
${ }^{15}$ Note that the optimal action conditional to the signal observed $a_{i}^{*}$ does not change with respect to the case where anticipatory utility only is maximized. In fact, here, the DM's FOC is $\left[u_{i}^{\prime}(\cdot)+1\right] \frac{\partial E\left[w-(w-a)^{2} \mid s_{i}\right]}{\partial a_{i}}=0$.
} 
Since a convex term has been added to the DM's objective function, Lemma 1 and Remark 6 (the latter in the appendix) must be slightly modified.

Corollary 1. The uninformative signal is a local minimum of total utility if

$$
R_{u}\left(f_{\frac{1}{2}}\right)<w_{1}+w_{2}-\frac{1}{2}\left(w_{1}-w_{2}\right)^{2}+\varepsilon
$$

where $\varepsilon \equiv \frac{2 f\left(\frac{1}{2} ; w_{1}, w_{2}\right)}{u^{\prime}\left(f\left(\frac{1}{2} ; w_{1}, w_{2}\right)\right)}$.

Note that $f\left(\frac{1}{2} ; w_{1}, w_{2}\right)$ and $\varepsilon$ are positive for $w_{1} \in\left(w_{2}+1-\sqrt{4 w_{2}+1}, w_{2}+1+\sqrt{4 w_{2}+1}\right)$. The following corollary is the equivalent of Remark 6 in the case of total utility, that is, it derives the condition such that total utility is decreasing in the signal precision for the fully informative signal.

Corollary 2. Total utility is decreasing in the signal precision for $q=1$ if and only if:

$$
\frac{u^{\prime}\left(w_{1}\right)}{u^{\prime}\left(w_{2}\right)}<\frac{1-\left(w_{1}-w_{2}\right)}{1+\left(w_{1}-w_{2}\right)}-\frac{2\left(w_{1}-w_{2}\right)}{u^{\prime}\left(w_{2}\right)\left[1+\left(w_{1}-w_{2}\right)\right]}
$$

Finally, the following corollary states sufficient conditions for an internal solution in the case of total utility:

Corollary 3. With total utility, if both the uninformative signal is a minimum of anticipatory utility and anticipatory utility is decreasing in the signal precision for the fully informative signal, that is if conditions 6.4 and 6.5 simultaneously hold, a partially informative signal is the DM's optimal choice.

Note that, with total utility, it is less likely that the objective function is decreasing in $q$ for the fully informative signal, however the condition such that the uninformative signal is a local minimum is less stringent than before.

To summarize, when the DM maximizes total utility and an internal solution exists, the latter is compatible with levels of information aversion higher than the values we observed when anticipatory utility was considered alone. Our simulations show that an internal solution with total utility can be found, for example when $\gamma=.56, w_{1}=.53$ and $w_{2}=.023$. 


\section{References}

[1] Barigozzi F, and R. Levaggi (2008). "Emotions in Physician Agency", Health Policy, 88, $1-14$.

[2] Caplin, A. and k. Eliaz (2003). "AIDS and Psychology: A Mechanism-Design Approach", RAND Journal of Economics, 34, 631-646.

[3] Caplin A, and J. Leahy (2001). "Psychological Expected Utility Theory and Anticipatory Feelings", Quarterly Journal of Economics, 55-80.

[4] Caplin, A. and J. Leahy (2004). "The Supply of Information by a Concerned Expert", Economic Journal, 114 , 487-505.

[5] Eliaz, k., Spiegler R. (2006). "Can Anticipatory Feelings Explain Anomalous Choices of Information Sources?", Games and Economic Behaviors, 56, 87-104.

[6] Jacobsen, Paul, Heiddis Valdimarsdottir, karen Brown, and kenneth O\&t, (1997). "DecisionMaking about Genetic Testing Among Women at Familial Risk for Breast Cancer," Psychosomatic Medicine, 59, 459-466

[7] karlsson N., G. Loewenstain and D. Seppi (2005). "The 'Ostrich Effect': Selective Exposure to Information About Investment", mimeo.

[8] kozsegi, B. (2003). "Health Anxiety and Patient Behavior", Journal of Health Economics, $22,1073-1084$.

[9] kozsegi, B, (2006). "Emotional Agency", Quarterly Journal of Economics,121(1): 121-155.

[10] Lerman, Caryn, M. Daly, M. Masny, and A. Balsheim, (1994). "Attitudes about Genetic Testing for Breast-Ovarian Cancer Susceptibility" Journal of Clinical Oncology, 12 843-850.

[11] Lerman, C., C. Hughes, S. J. Lemon, D. Main, C. Snyder, C. Durham, S. Narod and H. T. Lynch, (1998). "What You Don’t know Can Hurt You: Adverse Psychological Effects in Members of BRCA1-Linked and BRCA2-Linked Families Who Decline Genetic Testing", Journal of Clinical Oncology 16, 1650-1654.

[12] Quaid, k and M. Morris, (1993). "Reluctance to Undergo Predictive Testing: the Case of Huntington's Disease", American Journal of Medical Genetics, 45, 41-45. 
[13] Yariv, L., (2005). "I'll see it when I believe it - A simple model of Cognitive Consistency". Mimeo, UCLA. 


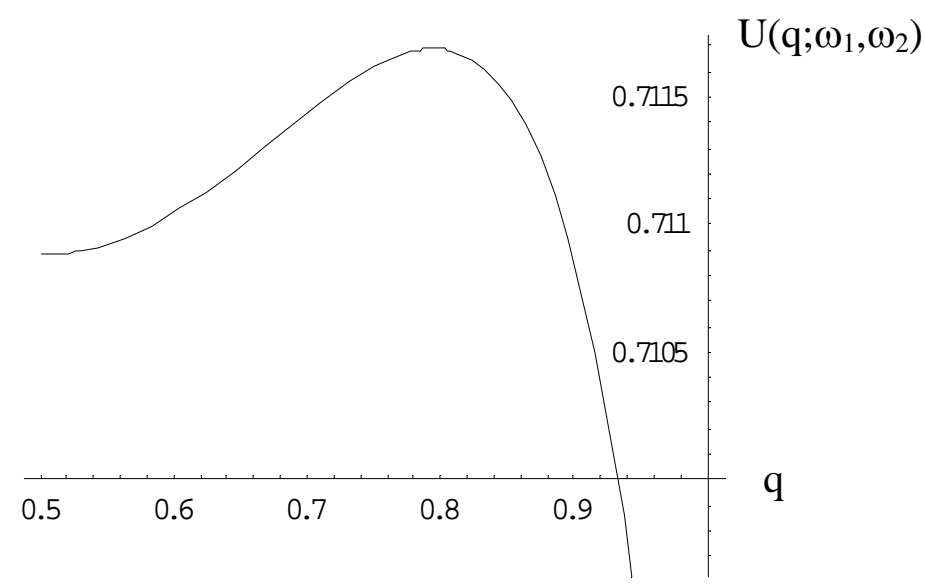

Figure 1: anticipatory utility using the power function with $\gamma=.42, \omega_{1}=.55$ and $\omega_{2}=.023$.

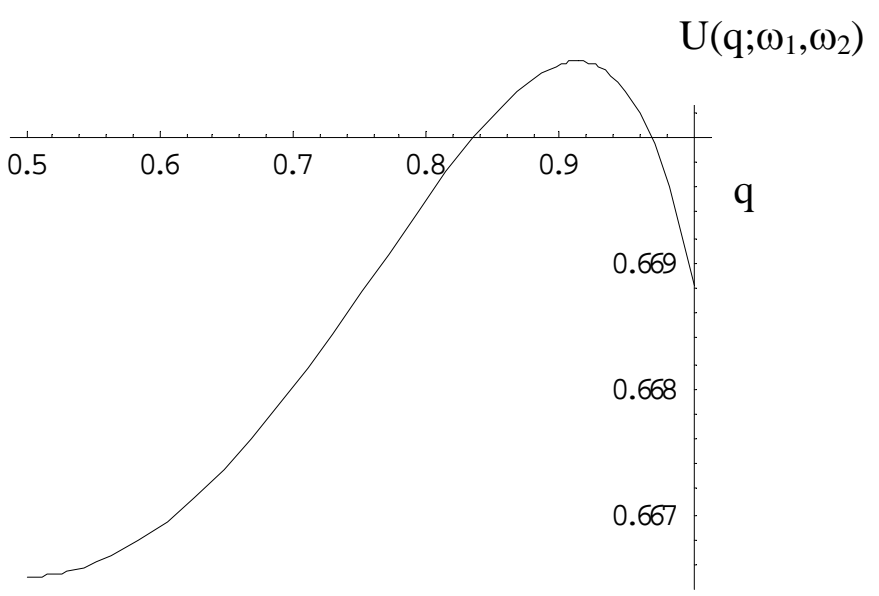

Figure 2: anticipatory utility using the power function with $\gamma=.4, \omega_{1}=.55$ and $\omega_{2}=.023$. 